\title{
Newton-type Methods for Solving the Nonsmooth Equations with Finitely Many Maximum Functions
}

\author{
Ruiying Wang, Shouqiang $\mathrm{Du}^{*}$ and Zhimin Liu \\ College of Mathematics and Statistics, Qingdao University, Qingdao, 266071. \\ Email: sqdu@qdu.edu.cn
}

\begin{abstract}
In this paper, we consider the Newton type methods for solving the nonsmooth equations with finitely many maximum functions. A new $\partial_{*}$-differential is used in the given Newton-type methods. The Newton-type methods also include a new parameterized combination. The superlinear convergence of the given methods is presented. Finally, the numerical experiments highlight the efficiency of the given methods.
\end{abstract}

Keywords: Nonsmooth equations, parameterized combinations, Newton method, superlinear convergence.

\section{Introduction}

In this paper, we consider the nonsmooth equations with finitely many maximum functions

$$
\begin{gathered}
\max _{j \in J_{1}} f_{1 j}(x)=0, \\
\vdots \\
\max _{j \in J_{n}} f_{n j}(x)=0,
\end{gathered}
$$

where $f_{i j}: R^{n} \rightarrow R$ for $j \in J_{i}, i=1, \cdots, n$ are assumed to be continuous differentiable, and $J_{i}, i=1, \cdots, n$ are finite index sets. Therefore, we know that (1.1) is a system of semismooth equations. As in [1], throughout the whole paper, we denote

$$
\begin{gathered}
f_{i}(x)=\max _{j \in J_{i}} f_{i j}(x), x \in R^{n}, i=1, \cdots, n, \\
F(x)=\left(f_{1}(x), \cdots, f_{n}(x)\right)^{T}, x \in R^{n}, \\
J_{i}(x)=\left\{j \in J_{i} \mid f_{i j}(x)=f_{i}(x)\right\}, x \in R^{n}, i=1, \cdots, n .
\end{gathered}
$$

And (1.1) can be transformed into the nonsmooth equations

$$
F(x)=0 .
$$

Obviously, $F$ is locally Lipschitzian and semismooth on $R^{n}$, see [1]. Many optimization problems and mathematical programming problems can be briefly rewritten as the form (1.2), e.g., $L C^{1}$ optimization problems $([2-5])$, nonlinear complementarity problems $([6])$ and variational inequality problems([7]).

Nonsmooth equation (1.2) was considered concurrently in [2] and [8]. One of the fundamental versions of the generalized Newton method was proposed by Qi and Sun in [2] as

$$
x_{k+1}=x_{k}-V_{k}^{-1} F\left(x_{k}\right),
$$

where $V_{k}$ is an element of Clarke generalized Jacobian [2], an element of B-differential [3] and an element of b-differential [9] of $F$ at $x_{k}$. And in [5], Chen and Qi presented the parameterized modification of generalized Jacobian Newton-like method

$$
x_{k+1}=x_{k}-\alpha_{k}\left(V_{k}+\lambda_{k} I\right)^{-1} F\left(x_{k}\right),
$$


where $V_{k} \in \partial_{B} F\left(x_{k}\right), I$ is the $n \times n$ identity matrix, $\alpha_{k}$ and $\lambda_{k}$ are chosen to ensure convergence and $V_{k}+\lambda_{k} I$ is invertible.

Recently, some research works have been conducted for solving (1.1). Specifically, Gao in [10] considered the Newton method for solving the problem (1.1), which is widely used in the optimal control, the variational inequality and complementarity problems, equilibrium problems, engineering mechanics $[8,11,12]$ and Karush-Kuhn-Tucker systems of nonlinear programming problems. Śmietański in [6] constructed the difference approximated Jacobian for a finite maximum function. A new smoothing nonlinear conjugate gradient method and a modified Levenberg-Marquardt method were also proposed for solving the nonsmooth equations (1.1) in [13] and [14]. Moreover, Śmietański considered the midpoint generalized Newton method in [15], in which a new approximation $x^{k+1}$ is

$$
x^{k+1}=x^{k}-\left(V_{x z}^{k}\right)^{-1} F\left(x^{k}\right), k=0,1, \cdots,
$$

where $V_{x z}^{k}$ is an element of some subdifferential of $F$ at $\frac{1}{2}\left(x^{k}+z^{k}\right)$ and $z^{k}=x^{k}-\left(V_{x}^{k}\right)^{-1} F\left(x^{k}\right), V_{x}^{k} \in$ $\partial_{B} F(x)$.

Based on the above research work, we present two new Newton-type methods with $\partial_{*}$-differential for (1.1). The $\partial_{*}$-differential for (1.1) is defined as

$$
\partial_{*} F(x)=\left\{\left(\nabla f_{1 j_{1}}, \cdots, \nabla f_{n j_{n}}\right)^{T} \mid j_{1} \in J_{1}(x), \cdots, j_{n} \in J_{n}(x)\right\}, x \in R^{n} .
$$

And the $\partial_{*} F(x)$ is a non-empty bounded set for each $x$ such that

$$
\partial_{*} F(x) \subset \partial f_{1}(x) \times \cdots \times \partial f_{n}(x),
$$

where $\partial f_{i}(x)$ is the Clarke generalized gradient of $f_{i}$ at $x$.

The remainder of the paper is organized as follows. In Section 2, we recall some proverbial results of generalized Jacobian, semismoothness and some propositions. In Section 3, we present a parameterized combinations Newton method for solving (1.1) and give the superlinear convergence of it. A modified parameterized combinations Newton method is also given to solve (1.1) and the local superlinear convergence is also proved. In Section 4, we report some numerical results of the two new Newton-type methods. In Section 5, we give some discussions to conclude this paper.

\section{Preliminaries}

If $F(x): R^{n} \rightarrow R^{n}$ is a locally Lipschitz function, and the limit

$$
\begin{aligned}
& \lim _{\substack{V \in \partial F\left(x+t h^{\prime}\right) \\
h^{\prime} \rightarrow h, t \rightarrow 0^{+}}} V h^{\prime} \\
&
\end{aligned}
$$

exists for any $h \in R^{n}$, then we say that $F$ is semismooth at $x$. As we all know, piecewise smooth functions and the maximum of a finite number of smooth functions are semismooth, which are introduced in $[2,10,15,16]$.

Proposition 2.1. Let $x \in R^{n}$. Suppose that for any $h \in R^{n}$

$$
\lim _{V \in \partial F(x+t h), t \downarrow 0} V h
$$

exists. Then

$$
F^{\prime}(x ; h)=\lim _{V \in \partial F(x+t h), t \downarrow 0} V h .
$$

Proposition 2.2. $F$ is semismooth at $x . \Longleftrightarrow$

$$
V h-F^{\prime}(x ; h)=o(\|h\|) .
$$


From [2], for any $h \rightarrow 0$,

$$
F(x+h)-F(x)-F^{\prime}(x ; h)=o(\|h\|),
$$

then we say $F$ is semismooth at $x$.

As [1], we give the following proposition.

Proposition 2.3. Suppose that $F$ is defined by (1.2). Then, for any $x, x^{*} \in R^{n}, V \in \partial F(x)$, we get

$$
F(x)-F\left(x^{*}\right)-V\left(x-x^{*}\right)=o\left(\left\|x-x^{*}\right\|\right) .
$$

Proof. By (2.1) and (2.2), we can obtain

$$
\begin{gathered}
V h-F^{\prime}\left(x^{*} ; h\right)=o(\|h\|), \forall V \in \partial F\left(x^{*}+h\right), \\
F\left(x^{*}+h\right)-F\left(x^{*}\right)-F^{\prime}\left(x^{*} ; h\right)=o(\|h\|) .
\end{gathered}
$$

Let $h=x-x^{*}$, then we obtain the proposition.

\section{The Newton-type Methods and Their Superlinear Convergence}

In this section, we present the new parameterized Newton methods for solving (1.1). Now we give the framework of the parameterized combinations Newton method.

Method 3.1(Parameterized Combinations Newton Method)

Step 0. Let $0 \leq \epsilon \leq 1, \alpha, \lambda \in(0,1), \lambda_{1}, \lambda_{2} \in[0,1], \lambda_{1}+\lambda_{2}=1, x^{0} \in R^{n}$. Let $k:=0$.

Step 1. If $\left\|F\left(x^{k}\right)\right\| \leq \epsilon$, then STOP.

Step 2. Find a point $y^{k} \in R^{n}$ such that

$$
y^{k}=x^{k}-\alpha\left(V_{x}^{k}+\lambda I\right)^{-1} F\left(x^{k}\right),
$$

where $V_{x}^{k} \in \partial_{*} F\left(x^{k}\right)$.

Step 3. Set

$$
x^{k+1}=x^{k}-\left(V_{x y}^{k}\right)^{-1} F\left(x^{k}\right), k=0,1, \cdots,
$$

where $V_{x y}^{k} \in \partial_{*} F\left(\lambda_{1} x^{k}+\lambda_{2} y^{k}\right)$. Let $k:=k+1$, go to Step 1 .

Remark 3.1. If $\lambda_{1}=\lambda_{2}=\frac{1}{2}$, the method we presented is equal to the case in [15].

Below we prove the convergence results of Method 3.1 with the $\partial_{*}$-differential. First of all, we need some helpful lemmas.

Lemma 3.2. Suppose that $F(x)$ and $\partial_{*} F(x)$ are defined by (1.2) and (1.3) respectively, and for any $V$, $V \in \partial_{*} F(x)$ is nonsingular. Then there exist $C>0, \gamma>0, \epsilon>0$ and $N(x, \epsilon)$ is a neighbor of $x$, such that

$$
\begin{gathered}
\left\|V^{-1}\right\| \leq C, \forall V \in \partial_{*} F(x), x \in N(x, \epsilon), \\
\|V\| \leq \gamma, \forall V \in \partial_{*} F(x), x \in N(x, \epsilon) .
\end{gathered}
$$

Lemma 3.3. Suppose that $x^{*}$ is the solution of (1.1). Then for any constants $\alpha, \lambda, \lambda_{2} \in(0,1)$, the function $G(x)=x-\left(\bar{V}_{x}\right)^{-1} F(x)$, where $\bar{V}_{x} \in \partial_{*} F\left(x-\alpha \lambda_{2}\left(V_{x}+\lambda I\right)^{-1} F(x)\right)$ and $V_{x} \in \partial_{*} F(x)$, is well-defined in a neighborhood of $x^{*}$.

Proof. By Lemma 3.1, there exists a scalar $C>0$ and a neighborhood $N$ of $x^{*}$ such that $V_{x}$ is nonsingular and $\left\|V_{x}^{-1}\right\| \leq C$ for any $x \in N(x, \epsilon)$ and $V_{x} \in \partial_{*} F(x)$. Firstly, let $\epsilon \in\left(0, \frac{1}{2 C}\right)$, we know that there exists a $V_{x^{*}} \in \partial_{*} F\left(x^{*}\right)$ such that for any $x \in S\left(x^{*}, \delta_{1}\right)$ and $V_{x} \in \partial_{*} F(x)$,

$$
\left\|V_{x}-V_{x^{*}}\right\|<\epsilon \text {. }
$$

Then, we consider $y=x-\alpha \lambda_{2}\left(V_{x}+\lambda I\right)^{-1} F(x)$, where $x \in N(x, \epsilon), V_{x} \in \partial_{*} F(x)$ and $\alpha, \lambda, \lambda_{2} \in(0,1)$. By the Corollary 3.2 in [3], it can be guaranteed that $y \in S\left(x^{*}, \delta_{1}\right)$, where $S\left(x^{*}, \delta_{1}\right)$ is an open ball in $R^{n}$ with center $x^{*}$ and radius $\delta_{1}$. Since $V_{y} \in \partial_{*} F(y)$, then (3.3) holds, i.e.

$$
\left\|V_{y}-V_{x^{*}}\right\|<\epsilon .
$$


So, let $\delta=\min \left\{\epsilon, \delta_{1}\right\}$, using the Banach perturbation lemma in [17], we obtain that $V_{x}$ is nonsingular and

$$
\begin{aligned}
\left\|\left(\bar{V}_{x}\right)^{-1}\right\| & =\left\|\left(V_{y}\right)^{-1}\right\|=\left\|\left[V_{x^{*}}+\left(V_{y}-V_{x^{*}}\right)\right]^{-1}\right\| \\
& \leq \frac{\left\|\left(V_{x^{*}}\right)^{-1}\right\|}{1-\left\|\left(V_{x^{*}}\right)^{-1}\right\|\left\|V_{y}-V_{x^{*}}\right\|} \\
& \leq \frac{C}{1-C \epsilon} \leq 2 C
\end{aligned}
$$

for $x \in S\left(x^{*}, \delta\right)$. Then, we get it.

Theorem 3.4. Suppose that $x^{*}$ is a solution of (1.1) and $\left\|V_{x^{*}}\right\| \leq \gamma$ for all $V_{x^{*}} \in \partial_{*} F\left(x^{*}\right)$ and all $V_{x^{*}}$ are nonsingular. Then there exists a neighborhood of $x^{*}$ such that the sequnence $\left\{x^{k}\right\}$ generated by Method 3.1 with $\partial_{*}$-differential converges superlinearly to $x^{*}$ for any initial point $x^{0}$ belonging to this neighborhood. Besides that, if $F\left(x^{k}\right) \neq 0$ for all $k$, then the norm of $F$ decreases superlinearly in a neighborhood of $x^{*}$, i.e.

$$
\lim _{k \rightarrow \infty} \frac{\left\|F\left(x^{k+1}\right)\right\|}{\left\|F\left(x^{k}\right)\right\|}=0 .
$$

Proof. By Lemma 3.1 and Lemma 3.2, for the first step $k=0$, the iterative formula (3.1) is well-defined in a neighborhood of $x^{*}$. And based on Lemma 3.2, we know that if $y=x-\alpha \lambda_{2}\left(V_{x}+\lambda I\right)^{-1} F(x)$, then we have $y \in S\left(x^{*}, \delta\right)$ and

$$
\begin{aligned}
\left\|x^{k+1}-x^{*}\right\| & =\left\|x^{k}-\left(V_{x y}^{k}\right)^{-1} F\left(x^{k}\right)-x^{*}\right\| \\
& =\left\|\left(V_{x y}^{k}\right)^{-1}\right\|\left\|F\left(x^{k}\right)-F\left(x^{*}\right)-V_{x y}^{k}\left(x^{k}-x^{*}\right)\right\| \\
& =o\left(\left\|x^{k}-x^{*}\right\|\right),
\end{aligned}
$$

where $V_{x y}^{k} \in \partial_{*} F\left(\lambda_{1} x^{k}+\lambda_{2} y^{k}\right), \lambda_{1}+\lambda_{2}=1$ and $\lambda_{1}, \lambda_{2} \in[0,1]$. Then the sequence $\left\{x^{k}\right\}(k \in N)$ is superlinearly convergent to $x^{*}$.

In the following we give the proof of (3.4). By Lemma 3.1, there exist $C>0$ and $\epsilon>0$ such that $V$ is nonsingular and $\left\|V^{-1}\right\| \leq C$ for any $x \in S\left(x^{*}, \epsilon\right)$ and $V \in \partial_{*} F(x)$. By Proposition 2.3, for any $\alpha \in(0,1)$, there is a $\delta_{2} \in(0, \epsilon)$ such that if $x \in S\left(x^{*}, \delta_{2}\right)$, we can see that

$$
\left\|F(x)-V\left(x-x^{*}\right)\right\| \leq \alpha\left\|x-x^{*}\right\| .
$$

And if $x^{k} \in S\left(x^{*}, \delta\right)$, we have

$$
\left\|x^{k+1}-x^{*}\right\| \leq \alpha\left\|x^{k}-x^{*}\right\|
$$

for $\delta \in\left(0, \delta_{2}\right)$. Since $\left\{x^{k}\right\}$ converges to $x^{*}$, there exists a $k_{\delta} \in N$ such that $\left\|x^{k}-x^{*}\right\| \leq \delta$ for all $k \geq k_{\delta}$. By (3.6), we have $\left\|x^{k+1}-x^{*}\right\| \leq \delta \leq \delta_{2}$. Furthermore, by (3.3), we have

$$
\left\|V_{x y}^{k+1}\right\|=\left\|V_{x y}^{k+1}-V_{x^{*}}+V_{x^{*}}\right\| \leq \epsilon+\left\|V_{x^{*}}\right\| \leq \epsilon+\gamma .
$$

By (3.5) and (3.6), we know that

$$
\begin{aligned}
\left\|F\left(x^{k+1}\right)\right\| & \leq\left\|V_{x y}^{k+1}\left(x^{k+1}-x^{*}\right)\right\|+\alpha\left\|x^{k+1}-x^{*}\right\| \\
& \leq(\epsilon+\gamma+\alpha)\left\|x^{k+1}-x^{*}\right\| \\
& \leq \alpha(\epsilon+\gamma+\alpha)\left\|x^{k}-x^{*}\right\| .
\end{aligned}
$$

By (3.1),(3.2) and (3.6), we obtain that

$$
\begin{aligned}
\left\|x^{k}-x^{*}\right\| & \leq\left\|x^{k+1}-x^{k}\right\|+\left\|x^{k+1}-x^{*}\right\| \\
& \leq\left\|\left(V_{x y}^{k}\right)^{-1} F\left(x^{k}\right)\right\|+\alpha\left\|x^{k}-x^{*}\right\| \\
& \leq C\left\|F\left(x^{k}\right)\right\|+\alpha\left\|x^{k}-x^{*}\right\| .
\end{aligned}
$$


Then

$$
\left\|x^{k}-x^{*}\right\| \leq \frac{C}{1-\alpha}\left\|F\left(x^{k}\right)\right\|
$$

Then we get

$$
\begin{aligned}
\left\|F\left(x^{k+1}\right)\right\| & \leq \alpha(\epsilon+\gamma+\alpha)\left\|x^{k}-x^{*}\right\| \\
& \leq \frac{C \alpha(\epsilon+\gamma+\alpha)}{1-\alpha}\left\|F\left(x^{k}\right)\right\| .
\end{aligned}
$$

Since $F\left(x^{k}\right) \neq 0$ for all $k$ and $\alpha$ may be arbitrarily small as $k \rightarrow \infty$, we get (3.4). Hence, we get this theorem.

Based on Method 3.1, in the following of this section, we establish a new iterator formula for solving (1.1). Given the $k$ th approximation $x^{k}$, the modification of the parameterized combinations Newton method obtains $x^{k+1}$ by means of

$$
x^{k+1}=x^{k}-\beta_{k}\left(V_{x y}^{k}+\mu_{k} I\right)^{-1} F\left(x^{k}\right), k=0,1, \cdots,
$$

where $V_{x y}^{k} \in \partial_{*} F\left(\lambda_{1} x^{k}+\lambda_{2} y^{k}\right), \lambda_{1}+\lambda_{2}=1, \lambda_{1}, \lambda_{2} \in[0,1]$ is an element of some subdifferential of $F$ at $\lambda_{1} x^{k}+\lambda_{2} y^{k}$ and $y^{k}=x^{k}-\alpha_{k}\left(V_{x}^{k}+\lambda_{k} I\right)^{-1} F\left(x^{k}\right)$, where $V_{x}^{k} \in \partial_{*} F\left(x^{k}\right)$.

Then we give the framework of the new modified method.

Method 3.2(Modified Newton Method)

Step 0. Let $0 \leq \epsilon \leq 1, \alpha, \beta, \lambda, \mu, \lambda_{1}, \lambda_{2} \in[0,1], \lambda_{1}+\lambda_{2}=1, x^{0} \in R^{n}$. Let $k:=0$.

Step 1. If $\left\|F\left(x^{k}\right)\right\| \leq \epsilon$, then STOP.

Step 2. Compute

$$
y^{k}=x^{k}-\alpha\left(V_{x}^{k}+\lambda I\right)^{-1} F\left(x^{k}\right),
$$

where $V_{x}^{k} \in \partial_{*} F\left(x^{k}\right)$.

Step 3. By (3.7), we obtain $x^{k+1}$. Let $k:=k+1$, go to Step 1 .

Remark 3.2. In Method 3.2, we replace the parameters $\beta_{k}$ and $\mu_{k}$ which are defined by (3.7) by $\beta \in(0,1], \mu \in[0,1]$, respectively.

The following theorem is the important result in this section and it also shows the local superlinearly convergence of the Method 3.2.

Theorem 3.5. Suppose that $x^{*}$ is a solution of (1.1), and all $V_{x^{*}} \in \partial_{*} F\left(x^{*}\right)$ are nonsingular. Let $\epsilon, \beta_{k}$, and $\mu_{k}$ satisfy $0<\beta_{1}<\beta_{k}<1, \epsilon<\gamma, 0<C\left(\epsilon\left(2+\beta_{1}\right)+\gamma\left(1-\beta_{1}\right)\right)<1$ and

$$
\left|\mu_{k}\right| \leq \mu^{\prime}<\frac{1-C\left(\epsilon\left(2+\beta_{1}\right)+\gamma\left(1-\beta_{1}\right)\right)}{2 C} .
$$

Then there exists a scalar $\delta>0$ such that for any $x^{0} \in S\left(x^{*}, \delta\right)$, the sequence $\left\{x^{k}\right\}$ defined by Method 3.2 is well-defined and converges linearly to $x^{*}$. Furthermore, if $\beta_{k} \rightarrow 1$ and $\mu_{k} \rightarrow 0$ as $k \rightarrow \infty$, then $\left\{x^{k}\right\}$ converges superlinearly to $x^{*}$.

Proof. By (2.3) and (2.4), there exists a constant $\delta>0$ such that for any $x \in S\left(x^{*}, \delta\right), V_{x} \in \partial_{*} F(x)$ and $h=x-x^{*}$, we have

$$
\begin{gathered}
\left\|V_{x}\left(x-x^{*}\right)-F^{\prime}\left(x^{*} ; x-x^{*}\right)\right\| \leq \epsilon\left\|x-x^{*}\right\|, \\
\left\|F(x)-F\left(x^{*}\right)-F^{\prime}\left(x^{*} ; x-x^{*}\right)\right\| \leq \epsilon\left\|x-x^{*}\right\| .
\end{gathered}
$$

By this formula together with (3.3), we obtain that

$$
\left\|V_{x}+\mu I-V_{x^{*}}\right\| \leq \epsilon+|\mu|<\frac{1}{C}
$$

when $|\mu|<\mu^{\prime}$. This implies that $V_{x}+\mu I$ is nonsingular and

$$
\left\|\left(V_{x}+\mu I\right)^{-1}\right\| \leq \frac{C}{1-C(\epsilon+|\mu|)} .
$$


Therefore (3.7) is well-defined for $x^{*} \in S\left(x^{*}, \delta\right)$. Furthermore, by (3.3) and $\left\|V_{x y}^{k}\right\| \leq \epsilon+\left\|V_{x^{*}}\right\| \leq \epsilon+\gamma$, we have

$$
\begin{aligned}
\left\|x^{k+1}-x^{*}\right\| & \leq\left\|\left(V_{x y}^{k}+\mu_{k} I\right)^{-1}\right\|\left(\beta_{k}\left\|F\left(x^{k}\right)-F\left(x^{*}\right)-F^{\prime}\left(x^{*} ; x^{k}-x^{*}\right)\right\|\right. \\
& \left.+\beta_{k}\left\|V_{x y}^{k}\left(x^{k}-x^{*}\right)-F^{\prime}\left(x^{*} ; x^{k}-x^{*}\right)\right\|+\left(\left(1-\beta_{k}\right)\left\|V_{x y}^{k}\right\|+\left|\mu_{k}\right|\right)\left\|x^{k}-x^{*}\right\|\right) \\
& \leq \frac{C}{1-C\left(\epsilon+\left|\mu_{k}\right|\right)}\left(2 \beta_{k}+\left(1-\beta_{k}\right)(\epsilon+\gamma)+\left|\mu_{k}\right|\right)\left\|x^{k}-x^{*}\right\| \\
& \leq \rho\left\|x^{k}-x^{*}\right\|,
\end{aligned}
$$

where $0<\rho=\frac{C}{1-C\left(\epsilon+\mu^{\prime}\right)}\left(2 \beta_{1} \epsilon+\left(1-\beta_{1}\right)(\epsilon+\gamma)+\mu^{\prime}\right)<1$. Hence $\left\{x^{k}\right\}$ defined by (3.7) converges linearly to $x^{*}$.

By

$$
\begin{gathered}
\left\|F(x)-F\left(x^{*}\right)-F^{\prime}\left(x^{*} ; x-x^{*}\right)\right\|=o\left\|x-x^{*}\right\|, \\
\left\|V_{x}\left(x-x^{*}\right)-F^{\prime}\left(x^{*} ; x-x^{*}\right)\right\|=o\left\|x-x^{*}\right\|,
\end{gathered}
$$

and let $\beta_{k} \rightarrow 1, \mu_{k} \rightarrow 0$ as $k \rightarrow \infty$, we get

$$
\left\|x^{k+1}-x^{*}\right\|=o\left(\left\|x^{k}-x^{*}\right\|\right) .
$$

Hence $\left\{x^{k}\right\}$ defined by (3.7) converges superlinearly to $x^{*}$, if $\beta_{k} \rightarrow 1, \mu_{k} \rightarrow 0$ as $k \rightarrow \infty$.

\section{Numerical Results}

In this part, we give some numerical results to illustrate the efficiency of the two given Newton-type methods. The Example 4.1 is based on the Example 4.1 in [14]. We compared Method 3.1 with fsolve which is taken from the Matlab optimization tool box. And for fsolve we choose "Algorithm to LevenbergMarquardt" and "Function tolerance to 1e-15". The numerical results are given in Table 4.1. The Example 4.3 and Example 4.4 are two semismooth equations from [15]. We compare the Method 3.1 and Method 3.2 with the midpoint Newton method [15], respectively, and give some numerical examples to illustrate the efficiency of the methods presented in our paper. The following notations are used: M1 is the Method 3.1; M2 is the Method 3.2; M3 is the midpoint Newton method introduced by Śmietański in [15]; $x^{0}$ is the initial point; $N$ is the number of iterations; $x^{k}$ is the final value after the iterative process. The symbol - denotes the test has failed. And the parameters used in this section are $\alpha=0.5, \beta=0.85, \lambda=0.5, \mu=0.125, \lambda_{1}=0.6, \lambda_{2}=0.4$ for all tests.

\section{Example 4.1.}

$$
\begin{aligned}
& \max \left\{f_{11}\left(x_{1}, x_{2}\right), f_{12}\left(x_{1}, x_{2}\right)\right\}=0, \\
& \max \left\{f_{21}\left(x_{1}, x_{2}\right), f_{22}\left(x_{1}, x_{2}\right)\right\}=0,
\end{aligned}
$$

where $f_{11}=x_{1}^{2}+x_{2}^{2}, f_{12}=x_{1}^{2}, f_{21}=\frac{1}{2}\left(x_{1}+2 x_{2}\right)^{2}, f_{22}=2\left(x_{1}+2 x_{2}\right)^{2}$. From (1.1), we know

$$
F(x)=\left(f_{1}(x), f_{2}(x)\right)^{T},
$$

where $f_{1}(x)=x_{1}^{2}+x_{2}^{2}, f_{2}(x)=2\left(x_{1}+2 x_{2}\right)^{2}, x \in R^{2}$. We know that $F(x)$ has one solution $(0,0)^{T}$. Then we compare M1 with fsolve in the final solution. The computational results are summarized in Table 4.1.

Table 4.1 


\begin{tabular}{c|c|c|c}
\hline & & $M 1$ & fsolve \\
\hline & $x^{0}$ & $x^{k}$ & $x^{k}$ \\
\hline 1 & $(-1,-1)^{T}$ & $(-3.324 e-011,9.249 e-011)^{T}$ & $(0,0)^{T}$ \\
2 & $(-2,-2)^{T}$ & $(-6.073 e-011,4.743 e-011)^{T}$ & $(0,0)^{T}$ \\
3 & $(-6,-3)^{T}$ & $(-4.994 e-011,6.755 e-011)^{T}$ & $(0,0)^{T}$ \\
4 & $(-5,-5)^{T}$ & $(-4.624 e-011,4.129 e-011)^{T}$ & $(0,0)^{T}$ \\
5 & $(1,0)^{T}$ & $(7.735 e-011,1.134 e-011)^{T}$ & $(0,0)^{T}$ \\
6 & $(1,1)^{T}$ & $(3.773 e-011,6.606 e-011)^{T}$ & $(0,0)^{T}$ \\
7 & $(2,3)^{T}$ & $(1.995 e-011,5.596 e-011)^{T}$ & $(0,0)^{T}$ \\
8 & $(10,10)^{T}$ & $(3.253 e-011,6.019 e-011)^{T}$ & $(0,0)^{T}$ \\
9 & $(-2,3)^{T}$ & $(-6.99 e-012,6.995 e-011)^{T}$ & $(0,0)^{T}$ \\
10 & $(-1,1)^{T}$ & $(-3.871 e-011,3.585 e-011)^{T}$ & $(0,0)^{T}$ \\
\hline
\end{tabular}

Example 4.2. Consider the nonsmooth equations with finitely many maximum functions given as (1.1). Similar as previously mentioned, we can obtain that

$$
F(x)=\left(f_{1}(x), f_{2}(x), \cdots, f_{9}(x)\right)^{T},
$$

where $f_{i}(x)=x_{i}^{2}+x_{i+1}^{2}, i=1, \cdots, 8, f_{9}(x)=x_{9}^{2}$. Obviously, this function has a unique solution $x=$ $(0,0,0,0,0,0,0,0,0)^{T}$. Then we compare $M 1$ with $M 3$ and fsolve, respectively. The numerical results are shown in Table 4.2 and Table 4.3.

Table 4.2

\begin{tabular}{c|c|c|c}
\hline & & $M 1$ & M3 \\
\hline & $x^{0}$ & $N$ & $N$ \\
\hline 1 & $(1,0,1,1,1,1,1,1,1)^{T}$ & 36 & $\times$ \\
2 & $(1,1,0,1,1,0,1,1,1)^{T}$ & 36 & $\times$ \\
3 & $(1,0,1,0,1,0,1,0,1)^{T}$ & 35 & $\times$ \\
4 & $(1,1,1,1,1,1,1,1,1)^{T}$ & 36 & 51 \\
5 & $(1,2,3,4,5,6,7,8,9)^{T}$ & 39 & 54 \\
\hline
\end{tabular}

Table 4.3

\begin{tabular}{c|c|c|c}
\hline & & $M 1$ & $f$ solve \\
\hline & $x^{0}$ & $x^{k}(e-11)$ & $x^{k}(e-15)$ \\
\hline 1 & $(1 ; 0 ; 1 ; 1 ; 1 ; 1 ; 1 ; 1 ; 1)$ & $(1.9 ; 0.3 ; 2.1 ; 2.1 ; 2.1 ; 2.1 ; 2.1 ; 2.2 ; 1.9)$ & $(0 ; 0 ; 0 ; 0 ; 0 ; 0 ; 0 ; 0 ; 0)$ \\
2 & $(1 ; 1 ; 0 ; 1 ; 1 ; 0 ; 1 ; 1 ; 1)$ & $(2.2 ; 1.9 ; 0.2 ; 2.2 ; 1.9 ; 0.4 ; 2.1 ; 2.2 ; 1.9)$ & $(0 ; 0 ; 0 ; 0 ; 0 ; 0 ; 0 ; 0 ; 0)$ \\
3 & $(1 ; 0 ; 1 ; 0 ; 1 ; 0 ; 1 ; 0 ; 1)$ & $(3.8 ; 0.9 ; 3.8 ; 0.9 ; 3.8 ; 0.9 ; 3.8 ; 0.9 ; 3.9)$ & $(0 ; 0 ; 0 ; 0 ; 0 ; 0 ; 0 ; 0 ; 0)$ \\
4 & $(1 ; 1 ; 1 ; 1 ; 1 ; 1 ; 1 ; 1 ; 1)$ & $(2.1 ; 2.1 ; 2.1 ; 2.1 ; 2.1 ; 2.1 ; 2.1 ; 2.1 ; 1.9)$ & $(0 ; 0 ; 0 ; 0 ; 0 ; 0 ; 0 ; 0 ; 0)$ \\
5 & $(1 ; 2 ; 3 ; 4 ; 5 ; 6 ; 7 ; 8 ; 9)$ & $(0.3 ; 0.7 ; 0.9 ; 1.4 ; 1.7 ; 2.2 ; 2.5 ; 3.1 ; 3.0)$ & $(0 ; 0 ; 0 ; 0 ; 0 ; 0 ; 0 ; 0 ; 0)$ \\
\hline
\end{tabular}

From Table 4.1 and Table 4.3, we know that Method 3.1 is similar to fsolve on the numerical accuracy. And from Table 4.2, we find that M1 needs less number of iterations than M3 in solving the same problem. Now, we give the following numerical examples to illustrate that Method 3.1 and Method 3.2 also adapt to semismooth equations with the Clarke generalized Jacobian, B-differential and b-differential.

Example 4.3. ([18])

$$
F(x)=\left(\begin{array}{l}
\left|x_{1}\right|+\left(x_{2}-1\right)^{2}-1 \\
\left(x_{1}-1\right)^{2}+\left|x_{2}\right|-1
\end{array}\right)=0 .
$$

The above problem is the system of semismooth equations and it has two solutions

$$
x^{*}=(0,0)^{T}, x^{* *}=(1,1)^{T} .
$$

The numerical results are shown in Table 4.4 and Table 4.5. 
Table 4.4

\begin{tabular}{c|cc|cc}
\hline & & $M 3$ & & $M 1$ \\
\hline$x^{0}$ & $N$ & $x^{k}$ & $N$ & $x^{k}$ \\
\hline$(0,1)^{T}$ & - & - & 14 & $(1.0000,1.0000)^{T}$ \\
$(0.5,0.5)^{T}$ & - & - & 15 & $(1.0000,1.0000)^{T}$ \\
$(0.5,2)^{T}$ & 33 & $(1.0000,1.0000)^{T}$ & 14 & $(1.0000,1.0000)^{T}$ \\
$(1,0)^{T}$ & - & - & 14 & $(1.0000,1.0000)^{T}$ \\
$(2,3)^{T}$ & 39 & $(1.0000,1.0000)^{T}$ & 24 & $(1.0000,1.0000)^{T}$ \\
$(10,10)^{T}$ & 38 & $(1.0000,1.0000)^{T}$ & 17 & $(1.0000,1.0000)^{T}$ \\
$(0,-1)^{T}$ & 33 & $(-1.086 e-011,-0.93 e-011)^{T}$ & 18 & $(-5.532 e-012,-5.533 e-012)^{T}$ \\
$(0,-10)^{T}$ & 39 & $(-5.143 e-011,-1.748 e-011)^{T}$ & 20 & $(1.0000,1.0000)^{T}$ \\
$(5,-6)^{T}$ & 37 & $(1.0000,1.0000)^{T}$ & 18 & $(1.0000,1.0000)^{T}$ \\
$(-1,0)^{T}$ & 33 & $(-9.334 e-011,-1.086 e-011)^{T}$ & 18 & $(-5.533 e-012,-5.532 e-012)^{T}$ \\
$(-10,0)^{T}$ & 39 & $(-1.748 e-011,-5.143 e-011)^{T}$ & 20 & $(1.0000,1.0000)^{T}$ \\
$(-1,-1)^{T}$ & 34 & $(-5.132 e-011,-5.132 e-011)^{T}$ & 18 & $(-1.094 e-011,-1.094 e-011)^{T}$ \\
$(-1.5,-1.5)^{T}$ & 35 & $(-3.847 e-011,-3.847 e-011)^{T}$ & 18 & $(-1.981 e-011,-1.981 e-011)^{T}$ \\
$(-5,-5)^{T}$ & 37 & $(-3.656 e-011,-3.656 e-011)^{T}$ & 20 & $(-9.158 e-012,-9.158 e-012)^{T}$ \\
$(-10,-10)^{T}$ & 38 & $(-4.373 e-011,-4.373 e-011)^{T}$ & 21 & $(-8.935 e-012,-8.935 e-012)^{T}$ \\
\hline
\end{tabular}

Table 4.5

\begin{tabular}{c|cc|cc}
\hline & & $M 3$ & $M^{2}$ \\
\hline$x^{0}$ & $N$ & $x^{k}$ & $N$ & $x^{k}$ \\
\hline$(0,1)^{T}$ & - & - & 35 & $(1.0000,1.0000)^{T}$ \\
$(0.5,0.5)^{T}$ & - & - & 34 & $(1.0000,1.0000)^{T}$ \\
$(0.5,2)^{T}$ & 33 & $(1.0000,1.0000)^{T}$ & 35 & $(1.0000,1.0000)^{T}$ \\
$(1,0)^{T}$ & - & - & 35 & $(1.0000,1.0000)^{T}$ \\
$(2,3)^{T}$ & 39 & $(1.0000,1.0000)^{T}$ & 37 & $(1.0000,1.0000)^{T}$ \\
$(10,10)^{T}$ & 38 & $(1.0000,1.0000)^{T}$ & 40 & $(1.0000,1.0000)^{T}$ \\
$(0,-1)^{T}$ & 33 & $(-1.086 e-011,-0.93 e-011)^{T}$ & 27 & $(4.799 e-011,2.966 e-011)^{T}$ \\
$(0,-10)^{T}$ & 39 & $(-5.143 e-011,-1.748 e-011)^{T}$ & 40 & $(1.0000,1.0000)^{T}$ \\
$(5,-6)^{T}$ & 37 & $(1.0000,1.0000)^{T}$ & 39 & $(1.0000,1.0000)^{T}$ \\
$(-1,0)^{T}$ & 33 & $(-9.334 e-011,-1.086 e-011)^{T}$ & 27 & $(-2.966 e-011,4.799 e-011)^{T}$ \\
$(-10,0)^{T}$ & 39 & $(-1.748 e-011,-5.143 e-011)^{T}$ & 40 & $(1.0000,1.0000)^{T}$ \\
$(-1,-1)^{T}$ & 34 & $(-5.132 e-011,-5.132 e-011)^{T}$ & 18 & $(-4.628 e-012,-4.628 e-012)^{T}$ \\
$(-1.5,-1.5)^{T}$ & 35 & $(-3.847 e-011,-3.847 e-011)^{T}$ & 18 & $(-9.293 e-012,-9.293 e-012)^{T}$ \\
$(-5,-5)^{T}$ & 37 & $(-3.656 e-011,-3.656 e-011)^{T}$ & 20 & $(-5.944 e-012,-5.944 e-012)^{T}$ \\
$(-10,-10)^{T}$ & 38 & $(-4.373 e-011,-4.373 e-011)^{T}$ & 21 & $(-7.788 e-012,-7.788 e-012)^{T}$ \\
\hline
\end{tabular}

Example 4.4. ([19])

$$
\begin{gathered}
f_{1}(x)=\left(x_{2}-x_{1}\right) \ln \left[\left(x_{2}-x_{1}\right)^{2}+1\right]+x_{2}-x_{1}, \\
f_{2}(x)= \begin{cases}-\exp \left(-x_{1}-x_{2}\right)+1, & \text { if } x_{2} \geq 0 \\
\frac{1-\exp \left(-x_{1}\right)}{1-x_{2}}, & \text { if } x_{2} \leq 0 .\end{cases}
\end{gathered}
$$

where the function $F: R^{2} \rightarrow R^{2}$ with component functions. And it has a unique solution $x^{*}=(0,0)^{T}$. And the results are shown in Table 4.6 and Table 4.7.

Table 4.6 


\begin{tabular}{c|cc|cc}
\hline & & $M 3$ & & M1 \\
\hline$x^{0}$ & $N$ & $x^{k}$ & $N$ & $x^{k}$ \\
\hline$(1,0)^{T}$ & 34 & $(5.932 e-011,6.46 e-012)^{T}$ & 20 & $(1.668 e-011,-1.031 e-011)^{T}$ \\
$(2,2)^{T}$ & - & - & 25 & $(-4.101 e-011,1.698 e-011)^{T}$ \\
$(-1,1)^{T}$ & 34 & $(-6.302 e-011,6.302 e-011)^{T}$ & 22 & $(-1.750 e-011,7.25 e-012)^{T}$ \\
$(-0.5,-0.5)^{T}$ & 33 & $(-6.338 e-011,-6.338 e-011)^{T}$ & 19 & $(-1.923 e-011,7.96 e-012)^{T}$ \\
$(-1,-1)^{T}$ & 34 & $(-5.493 e-011,-5.493 e-011)^{T}$ & 20 & $(-1.407 e-011,5.83 e-012)^{T}$ \\
$(-2,-2)^{T}$ & 35 & $(-5.116 e-011,-5.116 e-011)^{T}$ & 20 & $(-3.995 e-011,1.655 e-011)^{T}$ \\
$(-2,-3)^{T}$ & 35 & $(-5.331 e-011,-7.974 e-011)^{T}$ & 20 & $(1.031 e-011,-6.37 e-012)^{T}$ \\
$(-3,-3)^{T}$ & 36 & $(-4.458 e-011,-4.458 e-011)^{T}$ & 21 & $(-3.145 e-011,1.303 e-011)^{T}$ \\
$(5,-6)^{T}$ & - & - & 24 & $(-1.305 e-011,5.40 e-012)^{T}$ \\
$(-5,-6)^{T}$ & 37 & $(-6.517 e-011,-7.177 e-011)^{T}$ & 23 & $(-2.031 e-011,8.41 e-012)^{T}$ \\
\hline
\end{tabular}

Table 4.7

\begin{tabular}{c|cc|cc}
\hline & \multicolumn{2}{|c|}{ M3 } & & M2 \\
\hline$x^{0}$ & $N$ & $x^{k}$ & $N$ & $x^{k}$ \\
\hline$(1,0)^{T}$ & 34 & $(5.932 e-011,6.46 e-012)^{T}$ & 38 & $(-4.877 e-011,-7.892 e-011)^{T}$ \\
$(2,2)^{T}$ & - & - & 31 & $(2.465 e-011,5.952 e-011)^{T}$ \\
$(-1,1)^{T}$ & 34 & $(-6.302 e-011,6.302 e-011)^{T}$ & 31 & $(2.315 e-011,5.952 e-011)^{T}$ \\
$(-0.5,-0.5)^{T}$ & 33 & $(-6.338 e-011,-6.338 e-011)^{T}$ & 42 & $(-5.065 e-011,-8.196 e-011)^{T}$ \\
$(-1,-1)^{T}$ & 34 & $(-5.493 e-011,-5.493 e-011)^{T}$ & 43 & $(-6.47 e-011,-1.047 e-010)^{T}$ \\
$(-2,-2)^{T}$ & 35 & $(-5.116 e-011,-5.116 e-011)^{T}$ & 45 & $(-5.572 e-011,-9.015 e-011)^{T}$ \\
$(-2,-3)^{T}$ & 35 & $(-5.331 e-011,-7.974 e-011)^{T}$ & 45 & $(-7.06 e-011,-1.142 e-010)^{T}$ \\
$(-3,-3)^{T}$ & 36 & $(-4.458 e-011,-4.458 e-011)^{T}$ & 46 & $(-6.41 e-011,-1.037 e-010)^{T}$ \\
$(5,-6)^{T}$ & - & - & 48 & $(-6.18 e-011,-1.001 e-010)^{T}$ \\
$(-5,-6)^{T}$ & 37 & $(-6.517 e-011,-7.177 e-011)^{T}$ & 48 & $(-6.68 e-011,-1.080 e-010)^{T}$ \\
\hline
\end{tabular}

By Table 4.1 and Table 4.3, we obtain that Method 3.1 is efficient and it is similar to fsolve in the final solution, and Method 3.2 is invalid in Example 4.1 and Example 4.2. And Table 4.2, 4.4 and 4.6 indicate that M1 is promising since M1 need less number of iterations. From Table 4.5 and 4.7, we find that the result of M2 is similar to M3. But both M1 and M2 are promising. Especially when dealing with the problem of nonsingular matrix, our methods are more efficient than the midpoint Newton method given in [15].

\section{Conclusion}

Newton-type method is one of the most important tool for solving the nonsmooth equations with finitely many maximum functions, which is widely used in solving many economics, engineering and optimization problems. In this paper, we present two Newton-type methods with $\partial_{*}$-differential for solving the nonsmooth equations with finitely many maximum functions. And we prove the superlinear convergence of the given methods. Finally, we also show that the methods we propose are valid in semismooth equations with $\partial_{B}$-differential by the numerical experiments. As for the further work, we can also consider the global convergence of some methods to solve the problem of the nonsmooth equations with finitely many maximum functions.

Acknowledgments This work was supported by National Natural Science Foundation of China (NO. 11671220, 11401331), Natural Science Foundation of Shandong (No. ZR2015AQ013) and Key Issues of Statistical Research of Shandong Province (KT16276).

\section{References}

1. Y.Gao, "Newton methods for solving nonsmooth equations via a new subdifferential," Mathematical Methods of Operations Research, vol. 54, no. 2, pp. 239-257, 2001. 
2. L. Qi and J. Sun, "A nonsmooth version of Newton's method," Mathematical Programming, vol. 58, pp. 353-367, 1993.

3. L. Qi, "Convergence analysis of some algorithms for solving nonsmooth equations," Mathematics of Operations Research, vol. 18, pp. 227-244, 1993.

4. J. Marek and Śmietański, "On a new class parameterized Newton-like method for semismooth equations," Applied Mathematics and Computation, vol. 193, no. 2, pp. 430-437, 2007.

5. X. Chen and L. Qi, "A parameterized Newton method and a quasi-Newton method for nonsmooth equations," Computational Optimization and Applications, vol. 3, pp. 157-179, 1994.

6. J. Marek and Śmietański, "An approximate Newton method for non-smooth equations with finite max functions," Numerical Algorithms, vol. 41, pp. 219-238, 2006.

7. J. Pang and L. Qi, "Nonsmooth equations: motivation and algorithms," SIAM Journal on Optimization, vol. 3, pp. 443-465, 1993.

8. B. Kummer, "Newton's method based on generalized derivatives for nonsmooth functions: convergence analysis," Advances in Optimization, vol. 382, pp. 171-194, 1992.

9. D. Sun and J. Han, "Newton and quasi-Newton methods for a class of nonsmooth equations and related problems," SIAM Journal on Optimization, vol. 7, pp. 463-480, 1997.

10. Y. Gao, "Newton methods for solving two classes of nonsmooth equations," Applications of Mathematics, vol. 46, pp. 215-229, 2001.

11. L. Qi and P.Tseng, "On almost smooth functions and piecewise smooth functions," Nonlinear Analysis Theory Methods \& Applications, vol. 67, pp. 773-794, 2007.

12. F. Facchinei and JS. Pang, "Finite-Dimensional variational inequlities and complementarity problems," Springer, New York, 2003.

13. Y. Chen and S. Du, "A new smoothing nonlinear conjugate gradient method for nonsmooth equations with finitely many maximum functions," Abstract and Applied Analysis, 2013.

14. S. Du and Y. Gao, "A modified Levenberg-Marquardt method for nonsmooth equations with finitely many maximum functions," Mathematical Problem in Engineering, 2008.

15. J Marek and Śmietański, "Some quadrature-based versions of the generalized Newton method for solving nonsmooth equations," Journal of Computational and Applied Mathematics, vol. 235, pp. 5131-5139, 2011.

16. S. Du and Y. Gao, "A parameterized Newton method for vertical complementarity problem," OR Transactions, vol. 13, no. 1, pp. 22-28, 2009.

17. J.M. Ortega and W.C. Rheinboldt, "Iterative solution of nonlinear equations in several variables," Academic Press, New York, 1970.

18. H. Xu and W. Chang, "Approximate Newton methods for nonsmooth equations," Journal of Optimization Theory and Application, vol. 93, pp. 373-394, 1997.

19. M. Kojima and S. Shindo, "Extensions of Newton and quasi-Newton methods to systems of PC $C^{1}$ equations," Journal of the Operations Research Society of Japan, vol. 29, pp. 352-374, 1986. 